\title{
Mitteilungen der Schweizerischen Gesellschaft für Pathologie
}

Pathologe 2012 $33: 363$

DOI 10.1007s00292-012-1615-7

C) Springer-Verlag 2012
Schweizerische Gesellschaft für Pathologie

Dr. P. A. Diener, Sekretariat

Institut für Pathologie, Kantonsspital St. Gallen

Rorschacherstrasse 95

CH-9007 St. Gallen

Tel. 0041 (0)71 4942105

Fax. 0041 (0)71 4942894

E-Mail: P. A. Diener p.a.diener@kssg.ch

oderinfo@sgpath.ch

\section{Auflage der Qualitätsrichtlinien der Schweizer Gesellschaft für Pathologie}

Zwei Faktoren prägen die Entwicklung der Medizin in jüngster Zeit: Zum einen der beeindruckend rasante Wissensbzw. Erkenntniszuwachs, insbesondere im Bereich der biologischen bzw. genetischen Grundlagen verschiedener Erkrankungen. Zum anderen spielen Massnahmen zur Qualitätssicherung und Qualitätskontrolle eine immer grössere Bedeutung in allen Bereichen der Medizin. Von beiden ist auch - und insbesondere die Pathologie betroffen.

Vor diesem Hintergrund hat sich die Schweizer Gesellschaft für Pathologie (SGPath) entschlossen, Ihre Qualitätsrichtlinien in einer Neufauflage zu aktualisieren. Die letzte (geringfügige) Aktualisierung liegt beinahe 10 Jahre zurück. Die SGPath hat eine sog. „Qualitätskommission“. Diese hat für die aktualisierten Qualitätsrichtlinien die Funktion des Herausgebers übernommen. Aufgrund der kontinuierlichen Spezialisierung auch in der Pathologie kann kein Fachpathologe alle Gebiete so kompetent überblicken, wie es für die Verfassung der Leitlinien notwendig wäre. Daher wurden von den Herausgebern Autoren bzw. Autorenteams (Fachgruppenvertreter) gebeten, die Leitlinien ihrer jeweiligen Spezialgebiete zu verfassen.

Somit wurden alle Kapitel (gegliedert in einen allgemeinen und einen speziellen Teil) von Grund auf überarbeitet bzw. mehrheitlich vollständig neu geschrieben.

Darüber hinaus kamen mehrere Kapitel neu hinzu, was Ausdruck der oben erwähnten Entwicklungen in der Medizin bzw. in unserem Fach ist. Neu dazu gekommene Kapitel sind Molekularpathologie, juristische Rahmenbedingungen und Qualitätskontrolle im allgemeinen Teil sowie die Kapitel Haut, Leber, Plazenta, Knochenmark und endokrine Organe im organspezifischen Teil.

Die SGPath empfiehlt ihren Mitgliedern, sich bei ihrer täglichen Arbeit verbindlich an diese Richtlinien zu halten und lokale Traditionen oder persönliche Präferenzen zurückzustellen. Dies dient der Qualitätssicherung unserer Arbeit und dokumentiert diese auch gegenüber unseren Einsendern, klinischen Kollegen und insbesondere auch unseren Patienten.

Die 3. Auflage der Qualitätsrichtlinien, erschienen im 2011, wurde in einer deutschen und französischen Version erstellt. Neu ist auch, dass diese nun in elektronischer Form via die Homepage der SGPath (http:// www.sgpath.ch) zugänglich sind. Zusätzlich können selbstverständlich gegen einen Unkostenbetrag für die Druckkosten zusätzlich Versandspesen auch gebundene Exemplare beim Sekretär der SGPath bestellt werden.

\author{
Prof. Dr. Stephan Dirnhofer \\ (Vorsitzender der Kommission \\ Qualitätssicherung) \\ Institut für Pathologie, \\ Universitätsspitals Basel, \\ Schönbeinstrasse 40, 4003 Basel, \\ Schweiz \\ sdirnhofer@uhbs.ch
}

\section{Dr. Pierre-André Diener}

(Sekretär der SGPath)

Institut für Pathologie,

Kantonsspital St. Gallen,

9007 St. Gallen, Schweiz

p.a.diener@kssg.ch 